\title{
A GENERALISATION OF SUPERSOLUBLE GROUPS
}

\author{
BY
}

\section{R. J. HAGGARTY}

ABSTRACT. A $p$-soluble group $G$ belongs to the class $F(n, p)$ whenever the ranks of the $p$-chief factors of $G$ divide $n$ and $G$ has order coprime to $n$. A group in $F(n, p)$ is characterised by the embedding of its maximal subgroups. Whenever $N_{1}$ and $N_{2}$ are normal subgroups of $G$, of coprime indices in $G$, which lie in $F(n, p)$, then $G$ lies in $F(n, p)$ also. $F(n)$ denotes the intersection, taken over all primes $p$, of the classes $F(n, p)$. Simple groups all of whose proper subgroups lie in $F(n)$ are determined. Given an integer $n>2$, there exist an integer $m$ with the same prime divisors as $n$ and a soluble group $G$ such that $G$ lies in $F(m)$ but $G$ does not possess a Sylow tower. (We may take $m=n$ provided that $n$ is not a multiple of 1806 .) Furthermore, when $n$ is odd, an example of a soluble group $G$, all of whose proper subgroups lie in $F(n)$ but $G$ has no Sylow tower, is given.

1. Introduction. Let $G$ denote a finite $p$-soluble group and suppose that $L_{1}, \ldots, L_{t}$ denote the $p$-chief factors of $G . L_{i}$ is then an elementary abelian $p$-group of order $p^{d_{i}}$, say. Define the arithmetic p-rank of $G$ by

$$
\bar{r}_{p}(G)=\underset{i=1, \ldots, t}{1 . c . m .}\left\{d_{i}\right\} .
$$

For each integer $n \geqslant 1$ we define a class of $p$-soluble groups by

$$
F(n, p)=\left\{p \text {-soluble groups } G \mid(|G|, n)=1 \text { and } \bar{r}_{p}(G) \text { divides } n\right\} \text {. }
$$

If $G$ is now a soluble group and $\pi(G)$ denotes the prime divisors of $|G|$ then we may talk of the arithmetic rank, $\bar{r}(G)$, where

$$
\bar{r}(G)=\underset{p \in \pi(G)}{\text { 1.c.m. }}\left\{\bar{r}_{p}(G)\right\} .
$$

Thus we gain the classes $F(n)=$ \{soluble groups $G \mid(|G|, n)=1$ and $\bar{r}(G)$ divides $n$ \}. Clearly $F(n)=\bigcap F(n, p)$, where the intersection is over all primes $p . F(1)$ is precisely the class of supersoluble groups, and in this paper we compare and contrast some of the properties of a general $F(n)$ with the class of supersoluble groups.

The classes $F(n, p)$ were introduced by Huppert [4, VI.8.3], where it is shown

Received by the editors July 2, 1974.

AMS (MOS) subject classifications (1970). Primary 20D99; Secondary 20D10, 20 D40.

Key words and phrases. Supersoluble groups, saturated formations, Fitting class, Sylow tower.

Cupyright @ 1975. American Mathematical Society 
that $F(n, p)$ is a saturated formation. Since the local formations defining $F(n, p)$ are subgroup closed, we have that $F(n, p)$ is a subgroup closed class. Easily it follows that $F(n)$ is a subgroup closed saturated formation. In $\S 2$ we characterise a group in $F(n, p)$ by the embedding of its maximal subgroups. $F(n, p)$ is not a Fitting class; but if we insist that $G$ is a group possessing two normal subgroups $N_{1}$ and $N_{2}$ such that $N_{i} \in F(n, p)$ for $i=1,2$ and $N_{1}$ and $N_{2}$ have coprime in. dices in $G$, then indeed $N_{1} N_{2} \in F(n, p)$. This result constitutes $\S 3$. Corollary 3.2 generalizes the corresponding result for supersoluble groups provided by Friesen [2]. We say that a group $G$ is critical for a given class of groups if all proper subgroups of $G$ lie in the class but $G$ itself does not. In [1] Doerk investigates groups critical for supersoluble groups. These are soluble, and hence he is able to deduce many structure theorems for such groups. There exist simple groups which are critical for $F(n)$. We classify these in $\S 4$. In the final section, $\S 5$, we obtain results for groups critical for $F(2)$ similar to ones obtained by Doerk for groups critical for $F(1)$. One reason we are able to do so is that a group in $F(2)$ possesses an ordered Sylow tower. We conjecture that for $n>2$ there exist groups lying in $F(n)$ with no Sylow tower of any complexion. The rest of $\S 5$ is devoted to a contribution to this conjecture.

The author wishes to thank the referee for many helpful comments, particularly ones relating to the proof of 3.1 and the example at the end of $\S 5$.

2. A characterisation of $F(n, p)$. Let $H$ be a maximal subgroup of a finite $p$-soluble group, then the index $|G: H|$ of $H$ in $G$ is either a $p^{\prime}$-number or else it is a power of $p$.

THEOREM 2.1. $G \in F(n, p)$ if and only if $G$ is a finite p-soluble group of order coprime to $n$ and each maximal subgroup either has index $p^{k}$ for some in. teger $k$ which divides $n$, or has index coprime to $p$.

Proof. Firstly if $G \in F(n, p)$ and $N$ is a minimal normal subgroup of $G$ then either $N$ has order coprime to $p$ or else $N$ has order $p^{k}$ where $k$ divides $n$. Let $H$ be a maximal subgroup of $G$. If $N \leqslant H$ then $H / N$ is a maximal subgroup of $G / N$. Since $G / N \in F(n, p)$ we have by induction on $|G|$ that $H / N$ has the required index in $G / N$. But $|G: H|=|G / N: H / N|$ and so either $|G: H|$ is a $p^{\prime}$. number or else $|G: H|=p^{\alpha}$ where $\alpha$ divides $n$. Now suppose that $N \nless H$. By the maximality of $G$ we have that $G=H N$. Furthermore if $N$ is of $p^{\prime}$-order then $|G: H|=|H N: H|=|N: H \cap N|$ is $p^{\prime}$ also. We assume now that $N$ is of order $p^{k}$ where $k$ divides $n$. $H \cap N$ is normal in $H$ and since $N$ is now abelian $H \cap N$ is also normalised by $N$. Thus $G$ normalises $H \cap N$ and by the minimality of $N$ we conclude that $H \cap N=1$. Hence $|G: H|$ in fact equals $|N|$ and thus $H$ has index $p^{k}$ where $k$ divides $n$. 
Conversely suppose that $G$ is a $p$-soluble group of order coprime to $n$ with the stated restriction on its maximal subgroups. We show that $G \in F(n, p)$ by induction on $|G|$. If $\Phi(G)$, the Frattini subgroup of $G$, is nontrivial then since any maximal subgroup $H$ of $G$ contains $\Phi(G)$ we have that $H / \Phi(G)$ is a typical maximal subgroup of $G / \Phi(G)$. By induction $G / \Phi(G) \in F(n, p)$ and so $G \in$ $F(n, p)$ since $F(n, p)$ is a saturated class. When $\Phi(G)=1$, let $N$ be a minimal normal subgroup of $G$. Either $N$ has $p^{\prime}$-order or else $N$ is an elementary abelian $p$-group. In this latter case choose a maximal subgroup $H$ of $G$ which does not contain $N$. Then $G=H N$ and, as in the first paragraph of the proof, $|G: H|=$ $|N|$. Hence $N$ has order $p^{k}$ where $k$ divides $n$. Now $G / N \in F(n, p)$ by induction, and so we have shown that the chief factors of $G$ are either $p^{\prime}$ or else of $p$-power order, $p^{k}$ say, where $k$ divides $n$. Hence $G \in F(n, p)$.

COROllary 2.2. $G \in F(n)$ if and only if $G$ is a finite soluble group of order coprime to $n$ and each maximal subgroup of $G$ has prime power index, $p^{\alpha}$ say, where $\alpha$ is an integer dividing $n$.

RemarKs. (1) For $n=1$, Corollary 2.2 says that a soluble group $G$ is supersoluble if and only if all its maximal subgroups have prime index.

(2) Any group in which the maximal subgroups have index a prime or a prime squared is soluble. (See [4, VI. 9.4] due to Philip Hall.) This fact together with 2.2 for $n=2$ gives us the main result of Kohler [6].

(3) We conjecture that any group of order coprime to $n$ in which the maximal subgroups have prime power index with the exponent of the relevant prime dividing $n$ is soluble (and hence in $F(n)$ by 2.2). Denote this class by $X(n)$. Obviously our conjecture is true for $n=1,2$ and also for even $n$. At the end of $\S 4$ we are able to prove that the class $s)(n)$ consisting of those groups in $X(n)$ all of whose subgroups lie in $X(r)$ also consists solely of soluble groups. Thus our conjecture becomes: Is $X(n)=s \mathfrak{X}(n)$ ?

\section{A Fitting-type property of $F(n, p)$.}

THEOREM 3.1. Let $N_{1}$ and $N_{2}$ be normal subgroups of $G$ with $N_{i} \in F(n, p)$ for $i=1,2$. If $\left|G: N_{1}\right|$ and $\left|G: N_{2}\right|$ are coprime then $N_{1} N_{2} \in F(n, p)$.

Proof. Now $G=N_{1} N_{2}$ and so $G$ has order coprime to $n$ and we are required to show that all the $p$-chief factors of $G$ have order $p^{\alpha}$ for some integer $\alpha$ dividing $n$. Let $G$ be a minimal counterexample to the claim that $G \in F(n, p)$. Choose $M_{i}$ to be a maximal normal subgroup of $G$ containing $N_{i}$ for $i=1,2$. Now $M_{1}=\left(M_{1} \cap N_{2}\right) N_{1}$ and both $N_{1}$ and $M_{1} \cap N_{2}$ belong to $F(n, p)$. Also $\left|M_{1}: N_{1}\right|$ divides $\left|G: N_{1}\right|$ and $\left|M_{1}: M_{1} \cap N_{2}\right|=\left|N_{2} M_{1}: N_{2}\right|$ divides $\left|G: N_{2}\right|$; thus $N_{1}$ and $M_{1} \cap N_{2}$ are normal subgroups of $M_{1}$ of coprime indices. By the 
minimality of $G$ we conclude that $M_{1} \in F(n, p)$. In a similar fashion $M_{2} \in$ $F(n, p)$. Now $G=M_{1} M_{2}$ and so it suffices to prove our theorem when $M_{i}=N_{i}$ for $i=1,2$.

Let $N=N_{1} \cap N_{2}$; then if $N=1, G$ is the direct product of $N_{1}$ and $N_{2}$ and so lies in $F(n, p)$ and we are done. So choose $K$ to be a minimal normal subgroup of $G$ lying inside $N$. By the minimality of $G$ we have that $G / K \in F(n, p)$. If $K$ has $p^{\prime}$-order then $G \in F(n, p)$ and again we are finished. Hence $|K|=p^{k}$ for some integer $k$ and all we need to prove is that $k$ divides $n$ to contradict the existence of $G$ and so to prove our theorem. We divide the rest of the proof into several steps.

(a) If $L=C_{G}(K)$ then $L \leqslant N$. For suppose otherwise, then without loss of generality $L \nless N_{1}$. Now $G=L N_{1}$ and if $K_{1}$ is a minimal normal subgroup of $N_{1}$ with $K_{1} \leqslant K$ then certainly $N_{1}$ normalises $K_{1}$. Since $L$ centralizes $K$, it certainly normalises $K_{1}$. We deduce then that $K_{1} \triangleleft G$ and so $K_{1}=K$ and because $N_{1} \in F(n, p)$ we conclude that $k$ divides $n$ as required.

(b) $N_{i} / L$ is an abelian group for $i=1,2$. Let $K_{1}$ be a minimal subgroup of $N_{1}$ such that $K_{1} \leqslant K$. $K$ may be regarded as an irreducible $G$-module and by Clifford's theorem when $K$ is regarded as an $N_{1}$-module it is a direct sum of the conjugates of $K_{1}$. By assumption the dimension of $K_{1}$ is a divisor of $n$. By [4, VI. 8.1], $N_{1} / C$ is abelian where $C=C_{N_{1}}\left(K_{1}\right)$. Since $x^{-1} C x=C_{N_{1}}\left(x^{-1} K_{1} x\right)$, we deduce that $\bigcap_{x \in G}\left(x^{-1} C x\right)$ is equal to $L$. Hence $N_{1} / L$ is also abelian. Similarly $N_{2} / L$ is abelian.

(c) $\left|G: N_{i}\right|=q_{i}$ for $i=1,2$ where $q_{1}$ and $q_{2}$ are distinct primes. By (a) $L \leqslant N_{i}$ for $i=1,2$. Let $\bar{G}=G / L$ and $\bar{N}_{i}=N_{i} / L$ for $i=1,2$. Then $\bar{G}$ is the join of two normal subgroups $\bar{N}_{1}$ and $\bar{N}_{2}$ both of which are abelian by (b). Hence $G / N_{i} \cong \bar{G} / \bar{N}_{i}$ is abelian for $i=1,2$ so by the maximality of $N_{i}$ we deduce that $\left|G: N_{i}\right|=q_{i}$ is a prime. They are distinct since the indices are coprime.

(d) $G / L$ is abelian. Since $G=N_{1} N_{2}$ we deduce from (b) that $N / L$ is contained in the centre of $G / L$. Now $G / L / N / L \cong G / N$ is a direct product of two cyclic groups of orders $q_{1}$ and $q_{2}$ respectively. Thus $G / L$ is a cyclic extension of a central subgroup and is thus abelian.

(e) If $|N: L|=r$ then $|G / L|=q_{1} q_{2} r$ divides $\left(p^{k}-1\right)$ and moreover $k$ is minimal such that this occurs. As $L=C_{G}(K)$ we have that $G / L$ may be regarded as an abelian group of transformations of the vector space $K$ of dimension $k$ over $\mathrm{GF}(p)$. [4, II.3.10] applies to yield that $G / L$ is in fact cyclic of order dividing $\left(p^{k}-1\right)$ and also establishes the minimality of $k$.

(f) $q_{1} q_{2} r$ divides $\left(p^{n}-1\right)$. Let $K_{1}$ and $C$ be as in (b). Then [4, VI.8.1] yields further that $N_{1} / C$ is cyclic of order dividing $\left(p^{k_{1}}-1\right)$ where $\left|K_{1}\right|=p^{k_{1}}$. Since $k_{1}$ divides $n$ we see that $\left|N_{1} / C\right|$ divides $\left(p^{n}-1\right)$. But now $\left|N_{1} / x^{-1} C x\right|$ also divides $\left(p^{n}-1\right)$ for each $x \in G$. Hence the abelian group $N_{1} / L$ has exponent 
dividing $\left(p^{n}-1\right)$. By (e) $N_{1} / L$ is cyclic and so the order of $N_{1} / L$ divides $\left(p^{n}-1\right)$. That is to say $q_{2} r$ divides $\left(p^{n}-1\right)$, or equivalently $q_{2}$ divides $\left(p^{n}-1\right) / r$. Similarly $q_{1}$ divides $\left(p^{n}-1\right) / r$, but as $q_{1} \neq q_{2}$ and they are primes, we conclude that $q_{1} q_{2}$ divides $\left(p^{n}-1\right) / r$ and so $q_{1} q_{2} r$ divides $\left(p^{n}-1\right)$.

Finally then, by the minimality of $k$ from step (e) we deduce that $k$ divides $n$. This is our final contradiction.

Corollary 3.2. Let $N_{1}$ and $N_{2}$ be normal subgroups of $G$ with $N_{i} \in F(n)$ for $i=1$, 2. If $\left|G: N_{1}\right|$ is coprime to $\left|G: N_{2}\right|$ then $N_{1} N_{2} \in F(n)$.

4. Simple groups critical for $F(n)$. Let $G$ be a finite group critical for some $F(n)$; then all proper subgroups of $G$ are soluble. Thompson has classified such groups and they are

(i) $\operatorname{PSL}(2, p) ; p>3$ and $p^{2} \not \equiv 1 \bmod (5), p$ a prime.

(ii) $\operatorname{PSL}\left(2,3^{q}\right) ; q$ an odd prime.

(iii) $\operatorname{PSL}(3,3)$.

(iv) $\operatorname{PSL}\left(2,2^{q}\right) ; q$ a prime.

(v) The Suzuki groups, $\mathrm{Sz}\left(2^{q}\right) ; q$ odd.

Inspection of the subgroups of PSL $\left(2, p^{f}\right)$ given in [4, II. 8.27] yields that types (i) and (ii) involve $A_{4}$ which has $\bar{r}\left(A_{4}\right)=2$ and even order so cannot lie in any $F(n)$. The map

$$
\begin{gathered}
\phi: \mathrm{GL}(2,3) \rightarrow \operatorname{PSL}(3,3), \\
\left(\begin{array}{ll}
\alpha_{1} & \alpha_{2} \\
\alpha_{3} & \alpha_{4}
\end{array}\right) \mapsto\left(\begin{array}{lll}
\alpha_{1} & \alpha_{2} & 0 \\
\alpha_{3} & \alpha_{4} & 0 \\
0 & 0 & \epsilon
\end{array}\right)
\end{gathered}
$$

where $\epsilon=\alpha_{1} \alpha_{4}-\alpha_{2} \alpha_{3}$ is a monomorphism so $\operatorname{PSL}(3,3)$ has a subgroup isomorphic to $\operatorname{GL}(2,3)$ and the latter involves $A_{4}$. Thus types (i), (ii) and (iii) are not critical for any $F(n)$. We now prove

THEOREM 4.1. PSL $\left(2,2^{q}\right)$ for $q \geqslant 5$ is critical for $F(q)$. PSL $(2,4)$ and $\operatorname{PSL}(2,8)$ are not critical for any $F(n)$.

Proof. By [4, II. 8.27] the subgroups of $\operatorname{PSL}\left(2,2^{q}\right)$ are either supersoluble or of the following type: a semidirect product of an elementary abelian 2-group of order $2^{m}$ by a cyclic group of order $t$ where $t$ divides $\left(2^{m}-1\right)$ and $\left(2^{q}-1\right)$. Hence since all subgroups of $\operatorname{PSL}\left(2,2^{q}\right)$ cannot be supersoluble [1], we conclude that $m=q$. Let $P$ be a Sylow 2-subgroup of $\operatorname{PSL}\left(2,2^{q}\right)$ then $P$ has order $2^{q}$ and is elementary abelian. Let

$$
Z_{t}=g p\left\{\left(\begin{array}{ll}
\lambda & 0 \\
0 & \lambda^{-1}
\end{array}\right) \mid \lambda^{2^{t}}=1 \text { in } \mathrm{GF}\left(2^{q}\right)\right\}
$$


then the only nonsupersoluble subgroups of $\operatorname{PSL}\left(2,2^{q}\right)$ must be of the form $G=P$. $\mathbf{Z}_{\boldsymbol{t}}$. We prove that $\bar{r}(G)$ divides $q$.

Refine the series $1<P \leqslant G$ to a chief series for $G$. Since $G / P$ is cyclic all $2^{\prime}$. chief factors of $G$ have rank unity. Let $T_{i}$ be a 2-chief factor of $G$ and so $C_{i}=$ $C_{G}\left(T_{i}\right) \geqslant P$. Suppose that $\left|T_{i}\right|=2^{n_{i}}$ and $t_{i}=\left|G / C_{i}\right| . G / C_{i}$ may be realised as an irreducible subgroup of $\mathrm{GL}\left(n_{i}, 2\right)$ in the usual manner, and so [4, II.3.10] yields that $t_{i}$ divides $\left(2^{n_{i}}-1\right)$. Moreover $n_{i}$ is minimal such that this occurs. But $t_{i}$ divides $\left(2^{q}-1\right)$ and so $n_{i}$ must divide $q$. Hence $\bar{r}(G)$ divides $q$. Thus all proper subgroups of $\operatorname{PSL}\left(2,2^{q}\right)$ have arithmetic rank dividing $q$.

Now $\left|\operatorname{PSL}\left(2,2^{q}\right)\right|=2^{q}\left(2^{q}+1\right)\left(2^{q}-1\right)$. Since $2^{q} \equiv 2 \bmod (q)$ we deduce that $q$ divides $\left|\operatorname{PSL}\left(2,2^{q}\right)\right|$ if and only if $q=3$ or $q=2$. So for $q \geqslant 5$, $\operatorname{PSL}\left(2,2^{q}\right)$ is critical for $F(q)$. The group $\operatorname{PSL}(2,4)$ has a subgroup of even order with arithmetic rank 2. $\operatorname{PSL}(2,8)$ has a subgroup of order divisible by 3 with arithmetic rank 3. Our theorem now follows.

We now investigate type (v) groups. Suzuki [7] enumerates the subgroups of $\mathrm{Sz}\left(2^{q}\right)$. They are either supersoluble or else a Frobenius group $H$ of order $2^{2 q}\left(2^{q}-1\right)$. Moreover $H$ has a Frobenius kernel $Q$ of order $2^{2 q}$ and $H / Q$ is cyclic of order $\left(2^{q}-1\right)$. Thus all $2^{\prime}$-chief factors of $H$ are one dimensional. Now the Suzuki groups satisfy the condition that the centraliser of every nonidentity element is nilpotent. Hence our group $H$ also has this property. Let $T$ be a 2 . chief factor of $H$ (thus $T$ lies in $Q$ ). Then $C_{H}(T)$ is nilpotent and since $\operatorname{Fit}(H)$ is the intersection of the centralisers of all the chief factors of $G$ (see [4, VI.5.4]), we conclude that $\operatorname{Fit}(H)=C_{H}(T)$. Hence $C_{H}(T) \geqslant Q$ and so $H / C_{H}(T)$ is a cyclic group of order dividing $\left(2^{q}-1\right)$. If $|T|=2^{\alpha}$ then by [4, II.3.10] $\alpha$ is minimal such that $2^{\alpha} \equiv 1 \bmod \left(\left|H / C_{H}(T)\right|\right)$. Since $H$ is a Frobenius group, $H / Q$ fixes no nonidentity element of $Q$ and so $C_{H}(T)=Q$. But then $2^{q} \equiv 1 \bmod \left(\left|H / C_{H}(T)\right|\right)$. We conclude that $\alpha$ divides $q$. In fact $\alpha=q$ since not all subgroups of $\operatorname{Sz}\left(2^{q}\right)$ are supersoluble. Thus all proper subgroups of $\mathrm{Sz}\left(2^{q}\right)$ have arithmetic rank dividing $q$.

Now $\left|\operatorname{Sz}\left(2^{q}\right)\right|=2^{2 q}\left(2^{q}-1\right)\left(2^{2 q}+1\right)$ and since $2^{q} \equiv 2 \bmod (q)$ we deduce that $q$ divides the order of $\operatorname{Sz}\left(2^{q}\right)$ if and only if $q=5$ since $q$ is odd. We have thus proved

THEOREM 4.2. $\mathrm{Sz}\left(2^{q}\right)$ is critical for $F(q)$ for all odd primes $q \neq 5 . \mathrm{Sz}\left(2^{5}\right)$ has a subgroup of order 20 and so by the above cannot be critical for any $F(n)$.

We use the classification of this section to prove that if $G \in s \chi(n)$ then $G$ is is soluble. See $\S 2$, Remark (3). By induction on $|G|$ we have that all proper subgroups of $G$ are soluble. By $2.2, G$ is critical for $F(n)$. If $G$ possesses a nontrivial normal subgroup $N$ then induction yields that $G / N$ and $N$ are soluble. Hence $G$ is soluble. We are left with the case that $G$ is a simple group critical for $F(n)$. Hence by 4.1 and $4.2, G$ must be isomorphic to one of the following: 
(a) $\operatorname{PSL}\left(2,2^{q}\right)$ for $q$ a prime, $q \geqslant 5$.

(b) $\mathrm{Sz}\left(2^{q}\right)$ for $q$ an odd prime, $q \neq 5$.

But $\operatorname{PSL}\left(2,2^{q}\right)$ has a maximal subgroup of index $2^{q-1}\left(2^{q}+1\right)$ which is not even a prime power so $\operatorname{PSL}\left(2,2^{q}\right) \notin X(n)$. Also $\operatorname{Sz}\left(2^{q}\right)$ has a maximal subgroup of index $2^{2 q-1}\left(2^{2 q}+1\right)$ also not a prime power. Thus $G$ is neither a type (a) nor a type (b) group. We conclude that $G$ is soluble. Hence $s(n)=F(n)$.

5. Soluble groups critical for $F(n)$. To begin we deduce a structure theorem for groups critical for $F(2)$ similar to a corresponding result obtained by Doerk [1] for groups critical for $F(1)$. Firstly if $G$ is critical for $F(2)$ then $G$ is soluble. In fact either $G$ has odd order or else $G$ is cyclic of order 2 . We now assume that a group critical for $F(2)$ has odd order.

THEOREM 5.1. If $G$ is critical for $F(2)$ then there exists a unique prime $p$, dividing $|G|$, such that a Sylow p-subgroup of $G$ is normal in $G$. Furthermore the quotient $G / P$ has an ordered Sylow tower.

Proof. Any group of odd order with arithmetic rank dividing 2 possesses an order Sylow tower by [5, Satz 14]. Hence all proper subgroups of $G$ have an ordered Sylow tower. Let $r$ be the smallest prime divisor of $|G|$; then every proper subgroup of $G$ is $r$-nilpotent. Either $G$ itself is $r$-nilpotent or else $G$ is critical for the class of $r$-nilpotent groups. In the latter case a result of Ito (see for example [4, IV.5.4]) shows that in particular $G$ has the required structure. Hence we suppose that $G$ is $r$-nilpotent. Let $H=O_{r^{\prime}}(G)$; then $H \in F(2)$ and so $H$ possesses an ordered Sylow tower. Since $G / H$ is isomorphic to a Sylow $r$-subgroup of $G$ we conclude that $H$ and hence $G$ possesses a normal Sylow $p$-subgroup where $p$ is the largest prime dividing $|G|$. Let this be denoted by $P$.

$P$ is unique; for suppose that $Q$ is a normal Sylow $q$-subgroup of $G$ for some prime $q \neq p$. Then $G / P$ and $G / Q$ are isomorphic to Hall $p^{\prime}$ - and $q^{\prime}$-subgroups of $G$ respectively. Hence $G / P$ and $G / Q$ lie in $F(2)$. Hence $G=G / P \cap Q \in F(2)$, a contradiction.

Finally $G / P \in F(2)$ and so $G / P$ has an ordered Sylow tower.

By the complexion $\sigma$ of a Sylow tower we mean that a group $G$ has a Sylow tower with respect to some given ordering $\sigma$ of the primes dividing $|G|$. An ordered Sylow tower is then just the Sylow tower with respect to the natural ordering of the primes. Denote by $T$ the class of all groups which possess a Sylow tower of some complexion. Notice that if $G$ is critical for $F(2)$ then $G \in T$. We conjecture that if $n>2$ then there exist soluble groups critical for $F(n)$ which do not lie in $T$. In fact we assert that there exist soluble groups $G \in F(n) \backslash T$. Thus for $n>2$ we can hope for no structure theorem comparable to 5.1. We are able to prove 
THEOREM 5.2. Given an integer $n>2$ there exist an integer $m$ with the same prime divisors as $n$ and a soluble group $G$ with $G \in F(m) \backslash T$.

Proof. The "amazing prime theorem" (the origins of which we are uncertain) states that given an integer $n \geqslant 2$ and a prime $p$ then there exists a prime $q$ such that $n$ is the order of $p$ modulo $q$ unless either

(1) $n=2$ and $p=2^{q}-1$, or

(2) $p=2$ and $n=6$.

Let $p$ be the smallest prime not dividing $n>2$. Then we can find a prime $q$ such that $n$ is the order of $p$ modulo $q$ since the cases (1) and (2) above are ruled out by our choice of $n$ and $p$. By Fermat's theorem $q^{p-1} \equiv 1(\bmod p)$, and so if $\alpha$ is the order of $q$ modulo $p$ then $\alpha$ divides $p-1$. In particular $\alpha$ has the same prime divisors as $n$. We let $m=1$.c.m. $\{\alpha, n\}$, and so $m$ has the same prime divisors as $n$. We now construct a group $G$ with two prime divisors $p$ and $q$ which lies in $F(m)$ but not $T$.

Firstly notice that $p$ is coprime to $m$ by choice. Suppose that $q$ divides $m$, hence $q$ divides $n$. Hence writing $n=k q$ yields $p^{k q} \equiv 1(\bmod q)$ by the choice of $q$. But $p^{q} \equiv p(\bmod q)$ and so we deduce that $p^{k} \equiv 1(\bmod q)$ with $k<n$. This contradicts $n$ being the order of $p$ modulo $q$. Thus $q$ is also coprime to $n$ and hence $m$. So our group $G$ will have order coprime to $m$.

Let $G_{1}$ be the unique (up to isomorphism) nonnilpotent extension of an elementary abelian $p$-group of order $p^{n}$ by a cyclic group of order $q$. Let $G_{2}$ be the unique nonnilpotent extension of an elementary abelian $q$-group of order $q^{\alpha}$ by a cyclic group of order $p$. Finally let $G=G_{1} \times G_{2}$. By construction $\bar{r}\left(G_{1}\right)$ $=n$ and $\bar{r}\left(G_{2}\right)=\alpha$. Hence $G \in F(m)$. Hawkes [3] shows that $G$ is in fact critical for $T$. Thus $G \in F(m) \backslash T$.

Note. By using number theoretic techniques as above we can show that provided $n$ is not a multiple of $1806=2.3 .7 .43$ then there exists a soluble group $G \in F(n) \backslash T$.

Naturally if we cannot guarantee that a group in $F(n)$ necessarily has a Sylow tower then we cannot hope to generalise 5.1. The referee has kindly provided the following example.

EXAMPLE. Let $n$ be an odd integer, $n \geqslant 3$. Then there exists a finite soluble group $G$ which is critical for $F(n)$ but which does not have a Sylow tower of any complexion.

Firstly if $n$ is odd then there exists a prime number $q$ such that $n$ is the order of 2 modulo $q$. Let $D$ be the dihedral group of order $2 q$ generated by $x$ and $y$ where $x^{q}=y^{2}=1$ and $(x y)^{2}=1$. Let $V$ be a two-dimensional vector space over the field $\mathrm{GF}\left(2^{n}\right)$. The field $\mathrm{GF}\left(2^{n}\right)$ contains an element $\omega$ of multiplicative order $q$ since $q$ divides $\left(2^{n}-1\right)$. The following matrices define an 
irreducible representation of $D$ on $V$ :

$$
x \mapsto\left(\begin{array}{cc}
\omega & 0 \\
0 & \omega^{-1}
\end{array}\right), \quad y \mapsto\left(\begin{array}{ll}
0 & 1 \\
1 & 0
\end{array}\right) .
$$

From $G=V D$ the semidirect product of $V$ by $D$ with respect to the above action. $G$ has order $2^{2 n+1} q$ and clearly has no Sylow tower of any complexion. We show now that $G$ is critical for $F(n)$.

(a) $V$ is a minimal normal subgroup of $G$. $x$ fixes no elements of $V$ and so any subgroup of $V$ normalised by $x$ has order at least $2^{n}$. Thus $V$ is a direct sum of two subgroups $V_{1}$ and $V_{2}$ both of order $2^{n}$, and $V_{i}$ is normalized by $x$ for $i=$ 1 , 2. These subgroups afford irreducible representations of the cyclic group $\langle x\rangle$. These are inequivalent since $x$ acts as multiplication by $\omega$ on one and by $\omega^{-1}$ on the other. Hence $V_{1}$ and $V_{2}$ are the only proper subgroups of $V$ normalised by $x$. Now $y$ interchanges $V_{1}$ and $V_{2}$ hence $V$ is minimal normal as required.

(b) Let $M$ be a maximal subgroup of $G$; then either $V \leqslant M$ or else $M \cong D$. If $V$ is not contained in $M$ then $V \cap M \triangleleft G$ and by (a) $V \cap M=1$. Hence $D \cong$ $G / V=V M / V \cong M / V \cap M=M$ as required.

(c) All proper subgroups of $G$ belong to $F(n)$ but $G$ does not. Let $M$ be a maximal subgroup of $G$. If $M$ does not contain $V$ then $M \cong D$ by (b) and hence is supersoluble. If $M \geqslant V$ then either $M$ is a 2-group, which is supersoluble or else $M=\langle V, x\rangle$. By the proof of (a) we see that $\langle V, x\rangle \in F(n)$. Hence all proper subgroups of $G$ lie in $F(n)$. $G$ does not, since $\bar{r}(G)=2 n$.

\section{BIBLIOGRAPHY}

1. K. Doerk, Minimale nicht überauflösbare, endliche Gruppen, Math. Z. 91 (1966), 198-205. MR 33 \#189.

2. D. K. Friesen, Products of normal supersolvable subgroups, Proc. Amer. Math. Soc. 30 (1971), 46-48. MR 43 \#6310.

3. T. O. Hawkes, On the class of Sylow tower groups, Math. Z. 105 (1968), 393-398. MR 37 \#162.

4. B. Huppert, Endliche Gruppen I, Die Grundlehren der math. Wissenschaften, Band 134, Springer-Verlag, Berlin and New York, 1967. MR 37 \#302.

5. - Normalteiler und maximale Untergruppen endlicher Gruppen, Math. Z. 60 (1954), 409-434. MR 16, 332.

6. J. Kohler, Finite groups with all maximal subgroups of prime or prime square index, Canad. J. Math. 16 (1964), 435-442. MR 29 \#3533.

7. M. Suzuki, On a class of doubly transitive groups, Ann. of Math. (2) 75 (1962), 105-145. MR 25 \#112. POOL, ENGLAND 